BULLETIN Bulletin hispanique

HISPANIQUE Université Michel de Montaigne Bordeaux

119-1 | 2017

Autorité et pouvoir dans le théâtre du Siècle d'Or

\title{
Autorité et Pouvoir dans le théâtre de Cervantès
}

Quatre mises en scène du personnage du Roi

Jean Canavaggio

\section{OpenEdition}

Journals

Édition électronique

URL : http://journals.openedition.org/bulletinhispanique/4768

DOI : 10.4000/bulletinhispanique.4768

ISBN : 979-10-300-0142-6

ISSN : 1775-3821

Éditeur

Presses universitaires de Bordeaux

Édition imprimée

Date de publication : 15 juin 2017

Pagination : 79-88

ISBN : 979-10-300-0141-9

ISSN : 0007-4640

Référence électronique

Jean Canavaggio, "Autorité et Pouvoir dans le théâtre de Cervantès », Bulletin hispanique [En ligne], 119-1 | 2017, mis en ligne le 15 juin 2020, consulté le 11 septembre 2020. URL : http://

journals.openedition.org/bulletinhispanique/4768; DOI : https://doi.org/10.4000/bulletinhispanique. 4768

Tous droits réservés 


\title{
Autorité et Pouvoir dans le théâtre de Cervantès. Quatre mises en scène du personnage du Roi
}

\author{
Jean Canavaggio \\ Université de Paris X-Nanterre
}

Le présent article vise à montrer comment autorité et pouvoir tantôt s'opposent et tantôt convergent à travers quatre représentations du personnage du roi que nous propose le théâtre de Cervantès, dans El Trato de Argel, Los Baños de Argel, La Gran Sultana et Pedro de Urdemalas.

Mots-clés : Cervantès, roi, théâtre.

En este artículo se pretende mostrar cómo autoridad y poder ora se oponen ora coinciden en cuatro representaciones de la figura del rey que nos propone el teatro de Cervantes, en El Trato de Argel, Los Baños de Argel, La Gran Sultana y Pedro de Urdemalas.

Palabras clave: Cervantes, rey, teatro.

Based on the study of four representations of the figure of the king in Cervantès' theatre: "El Trato de Argel", "Los Baños de Argel", "La Gran Sultana" and "Pedro de Urdemalas", this article aims at demonstrating how authority and power can be seen as forces, sometimes contrasting sometimes convergent.

Keywords: Cervantes, king, theatre. 
utorité et pouvoir, parfois tenus pour synonymes, ne revêtent pas - exactement la même signification. Alors que le pouvoir existe dès lors qu'il dispose des moyens d'action qui en permettent l'exercice, indépendamment de la question de sa légitimité, l'autorité est fondée sur une supériorité de mérite ou de séduction qui est la condition d'une obéissance admise sans contrainte. Il en résulte qu'il y a autorité quand un pouvoir bénéficie d'un capital de confiance et quand les individus sur qui s'exerce le pouvoir lui conservent leur confiance. Ce pouvoir est alors perçu comme légitime et il acquiert l'autorité de ce qui échappe à la contestation.

Le rapport qui s'établit entre pouvoir et autorité s’inscrit par conséquent dans une dialectique susceptible de générer des tensions dont le théâtre a saisi très tôt le parti qu'il pouvait en tirer. On sait comment, en Espagne, la Comedia nueva en a exploité les possibilités, en particulier en opposant l'impartialité du roi justicier à l'arbitraire du despote. Avant même les débuts de Lope de Vega, les poètes de la génération de 1580 - Lupercio Leonardo de Argensola, Cristóbal de Virués, Juan de la Cueva - lui ont ouvert la voie en inscrivant la figure du tyran au cour de leur dramaturgie. Tout en partageant certaines de leurs préoccupations, Cervantès, leur contemporain, s'en sépare cependant dans le traitement qu'il réserve à ce personnage. Dans les pièces librement inspirées par son expérience de captif - El Trato de Argel, Los Baños de Argel et La Gran Sultana - c'est à une figure exotique, pacha ou sultan, qu'incombe cet exercice, mais selon des modalités qui ne se ramènent pas à un seul et même canon. Toutes trois, qui plus est, se distinguent de ce point de vue de Pedro de Urdemalas, où le personnage du roi nous apparaît sous un jour insolite qui lui assure une incontestable originalité.

El Trato de Argel et Los Baños de Argel, deux œuvres nourries des souvenirs de l'auteur, semblent accorder un rôle de second plan à Hassan Pacha, appelé « roi d'Alger ", mais qui tenait en fait son autorité du Sultan et était nommé pour une durée limitée ${ }^{1}$. Dans la première de ces deux pièces, il faut attendre la dernière scène de la quatrième journée pour le voir apparaître sur scène ; et, dans Los Baños de Argel, il ne se manifeste qu’à deux reprises : dans la première journée, lors de la réception réservée au corsaire Cauralí, au retour de la descente sur les côtes d'Espagne qui ouvre l'action ; puis, au milieu de la troisième journée, avant que ne s'évadent les captifs chrétiens emmenés par Don Lope et Zahara. Toutefois, ce choix ne revêt pas dans les deux cas la même valeur. Dans El

1. Sur le destin historique de ce renégat vénitien, pacha d'Alger de 1577 à 1580 et de 1582 à 1585, cf. Jaime Oliver Asín, «La hija de Agi Morato en la obra de Cervantes», BRAE, 27 (19471948), p. 281-287, ainsi que Emilio Sola et José F. de la Peńa, Cervantes y la Berbería (Cervantes, mundo turco-berberisco y servicios secretos en la época de Felipe II), México, Fondo de Cultura Económica, 1995, p. 132-155 et 218-275. Dans le récit du Captif (Don Quichotte, I, 40), Hassan est présenté comme «el más cruel renegado que jamás se ha visto». À l'époque de la captivité de Cervantès, le pacha était contraint, qui plus est, de composer avec les deux factions - la confrérie des corsaires (ou taifa des rä̈s) et la milice de janissaires (ou odjaq) - qui se disputaient la réalité du pouvoir dans la cité barbaresque. Cf. à ce sujet Fernand Braudel, La Méditerranée et le monde méditerranéen à l'époque de Philippe II, Paris, A. Colin, 1966, t. II, p. 205-208. 
Trato de Argel, qui date de la première époque du théâtre de Cervantès, le monde de la captivité s'ordonne autour du quatuor formé par Aurelio, Silvia et leurs maîtres respectifs. Il revient à ces quatre personnages d'animer une double intrigue amoureuse de pure invention qui se développe en dehors de toute intervention du roi d'Alger. C'est sur un autre souverain, Philippe II, que se porte l'attention du spectateur : à la faveur des propos qu'ils échangent à la fin de la première journée, Aurelio et Saavedra, porte-parole du poète, accueillent avec une heureuse surprise une rumeur, celle du rassemblement d'une armée espagnole. La progression de l'intrigue se trouve alors suspendue, tandis que l'arrière-plan historique vient occuper l'espace de la fiction qui se réorganise autour de la figure du Roi prudent. Invoqué, puis interpellé in absentia par Saavedra, Philippe II se voit invité à se lancer sans tarder dans une expédition militaire, la prise d'Alger, que Charles Quint, son père, avait vainement tentée quarante ans plus tôt :

Cuando me vea en más seguro estado,
y si la suerte o si el favor me ayuda
a verme ante Filipo arrodillado,
mi lengua balbuciente y casi muda
pienso mover en la real presencia
de adulación y de mentir desnuda,
diciendo : "Alto señor, cuya potencia
sujetas trae las bárbaras naciones
................................
despierte en tu real pecho coraje
la desvergüenza con que una bicoca
aspira de contino a hacerte ultraje...

Toutefois, la perspective ainsi ouverte par Saavedra relève d'un simple vœu pieux : les troupes réunies en Estrémadoure étaient en effet destinées, non pas à s'emparer de la ville, mais à occuper le Portugal au moment où venait d'être proclamée, au bénéfice de l'Espagne, la réunion des deux couronnes péninsulaires. Quant à l'expédition d'Alger, opération humanitaire sans réelle valeur stratégique, elle avait été mise en sommeil dès les lendemains de Lépante au profit d'autres aventures militaires, comme la prise de Tunis par Don Juan d'Autriche, avant d'être abandonnée sans rémission ${ }^{3}$.

Afin de maintenir l'intérêt du spectateur, cet abandon n'est nulle part évoqué dans la pièce. Néanmoins, dès lors que les espoirs exprimés par Saavedra ne reçoivent pas de confirmation immédiate, l'intrigue se détache de tout référent historique jusqu'à l'apparition finale du roi d'Alger. Celle-ci obéit jusqu'à un certain point à la logique interne de l'action, étant donné qu'Yzuf, le maitre de Silvia et d'Aurelio, a acheté la jeune femme sans en référer à Hassan, si bien que ce dernier, apprenant la chose, le convoque impérativement. Mais cette

2. El Trato de Argel, jornada 1a, vv. 414-421 et 426-428, dans Cervantes, Teatro completo, ed. Antonio Rey Hazas y Florencio Sevilla, Barcelona, Planeta, 1987, p. 856.

3. Cf. Braudel, La Méditerranée..., t. II, p. 384 et ss. 
convocation est également liée aux préparatifs militaires évoqués plus haut : Yzuf, en effet, bien qu'il ait reçu l'ordre de renforcer sans tarder les remparts de la ville ${ }^{4}$, s'est dérobé à sa mission. Aussi comparaît-il devant Hassan, qui exige alors qu'il lui remette Silvia. Devant le refus qui lui est opposé, le roi fait éclater sa fureur et le condamne à une bastonnade infamante :

$$
\begin{aligned}
& \text { - Al esclavo te doy, rey, sin dinero, } \\
& \text { y déjame la esclava, por quien muero. } \\
& \text { - ¿Tal osaste decir, oh moro infame? } \\
& \text { Llevalde abajo y dalde tanto palo, } \\
& \text { hasta que con su sangre se derrame } \\
& \text { el deseo que tiene torpe y malo }
\end{aligned}
$$

Dans cette scène, Hassan apparaît donc comme le type même du potentat qui s'abandonne à ses passions au lieu de se laisser guider par la raison; ce que confirme le châtiment qu'il inflige quelques instants plus tard à un esclave qui avait tenté en vain de s'évader :

$$
\begin{aligned}
& \text { - ¿Oh yuraja caur! Dalde seiscientos palos } \\
& \text { en las espaldas muy bien dados, } \\
& \text { y luego le daréis otros quinientos } \\
& \text { en la barriga y en los pies cansados. } \\
& \text { - ¿Tan sin razón ni ley tantos tormentos } \\
& \text { - Cito cifuti breguedi, jatalde, } \\
& \text { abrilde, desollalde y aun matalde! }
\end{aligned}
$$

Cependant, au moment même où le pacha condamne le malheureux au plus cruel des sorts, tout en multipliant les imprécations contre l'obstination de « ces chiens d'Espagnols ", le courage indomptable dont ces derniers font preuve en toutes circonstances le porte, sans transition, à exprimer son admiration pour les captifs de rançon qui, dit-il, ne manquent jamais à la parole donnée :

$$
\begin{aligned}
& \text { Una virtud en ellos he notado: } \\
& \text { que guardan su palabra sin reveses, } \\
& \text { y en esta mi opinión me han confirmado } \\
& \text { dos caballeros Sosas portugueses. } \\
& \text { Don Francisco también la ha sigurado } \\
& \text { que tiene sobrenombre de Meneses, } \\
& \text { los cuales sobre su palabra han sido } \\
& \text { enviados a España, y la han cumplido. } \\
& \text { Don Fernando de Ormaza también fuese } \\
& \text { sobre su fe y palabra, y ansí ha hecho } \\
& \text { un mes antes que el término cumpliese, } \\
& \text { la paga, con que bien me ha satisfecho. } \\
& \text { De darles libertad, un interese }
\end{aligned}
$$

4. Op cit., jornada 3a, vv. 1929-1940, p. 898-899.

5. Ibid., vv. 2228-2333, p. 909-910.

6. Ibid., vv. 2346-2352, p. 910-911. « O yuraja caur!» pourrait signifier : «Oh, chef chrétien!». "Cito cifuti breguedi» semble vouloir dire: "Arrête et tais-toi, espèce de juif». 
se sigue tal, que dobla mi provecho:

que como van sobre su fe prendados,

les pido los rescates tresdoblados ${ }^{7}$.

Ce brusque revirement atteste, sans aucun doute, la maladresse d'un dramaturge débutant qui, à en juger par les exemples que donne Hassan pour justifier sa conduite, a ressenti le besoin de faire appel à ses souvenirs de captivité $^{8}$; mais c'est aussi pour lui une façon de préparer la scène finale, qui voit Aurelio comparaître devant le roi. Celui-ci devient alors le contretype du tyran qu'il semblait être auparavant, pour assumer le rôle providentiel d'un deus ex machina: en promettant à Aurelio de le libérer, puis, en libérant sous parole le couple des amants, il s'identifie de la sorte à l'archétype du roi justicier. Certes, du point de vue d'un personnage qui reconnaît être mû avant tout par l'intérêt, il ne s'agit là que d'un pari :

\section{De pérdida y ganancia es este juego?.}

Cervantès, cependant, ne nous laisse pas sur cet aveu. Tout d'abord, l'interlocuteur de Hassan, un maure anonyme, souligne en termes sentencieux la portée de ce geste ;

Señor, del bien hacer siempre se espera
galardón, y si falta deste suelo,
la paga se dilata por el cielo ${ }^{10}$.

Puis, Aurelio en personne, dans son action de grâces finale, attribue à Dieu et à la Vierge ce revirement miraculeux, faisant ainsi du pacha l'instrument d'une décision du Ciel.

La vision qui nous est ainsi offerte s'altère sensiblement dans Los Baños, l'une des pièces incluses par Cervantès dans le recueil des Ocho Comedias, à ceci près que cette réélaboration affecte moins les caractéristiques du personnage que le rôle qui lui est dévolu. Comme il a été dit plus haut, il revient à Hassan d'accueillir le corsaire Cauralí à son retour au port. En ce sens, le pacha consacre la course en tant que source essentielle de la prospérité d'Alger, de même que la prééminence de la corporation des corsaires - ou taifa des raïs - au sein de la cité, au détriment du corps des janissaires. En même temps, il cautionne, par sa présence, une pratique aussi cruelle qu'arbitraire : les descentes opérées le long des côtes espagnoles du Levant, grâce à la complicité de morisques renégats originaires des villages mis à sac. Or, parmi les captifs que l'on débarque et qui vont être amenés au marché pour y être vendus, figurent deux enfants, Juanico

7. Ibid., vv. 2362-2377, p. 911.

8. Pour une identification des captifs évoqués dans cette tirade, cf. notre article "Un compagnon de captivité de Cervantès: don Fernando de Ormaza», Mélanges de la Casa de Velázquez, 2 (1966), p. 339-343.

9. Op. cit., jornada $4^{\text {a }}$, vv. 2382 , p. 912.

10. Ibid., vv. 2383-2385, p. 912. 
et Francisquito, qui se trouvent être les propres neveux d'un de ces renégats, appelé Yzuf. À la fin de la première journée, le meurtre d'Yzuf, perpétré par Hazén, un autre renégat qui, lui, veut retourner dans le giron de l'Église, confère une portée spectaculaire au juste châtiment de l'auteur d'un tel forfait.

Lorsqu'il reparaît sur scène, dans le cours de la troisième journée, Hassan retrouve le profil qui était le sien dans la séquence du Trato que nous avons analysée plus haut : après s'en être pris à la «canalla terca " des esclaves espagnols,

\section{porfiada, feroz, fiera y arrogante, pertinaz, indomable y atrevida ${ }^{11}$,}

il fait comparaître deux chrétiens capturés par les Maures alors qu'ils tentaient de s'enfuir, l'un par mer, l'autre par voie de terre. En même temps que les deux fugitifs se présente un troisième captif, le sacristain Tristán, dont les bouffonneries visent à introduire dans l'action une note comique. Ce personnage apparaît en compagnie d'un juif dont il vient de voler l'enfant. À la question du cadi - "¿Para qué quiere este niño?" - Tristán répond par une plaisanterie :

\section{Para que le rescaten, si no quieren que le crie y enseñe el Padrenuestro ${ }^{12}$.}

Et, tandis que le pacha lui ordonne de rendre l'enfant à son père, ce dernier reçoit du cadi l'ordre de débourser quarante aspres pour les donner au sacristain, "que los merece» ${ }^{13}$.

Ainsi, c'est à Hassan qu'il incombe de mener à son terme l'intrigue animée par le sacristain ; mais son intervention se trouve du même coup réduite au dénouement de cette action épisodique, sans pour autant affecter les deux intrigues principales qui alternent au fil de l'action : d'un côté, les amours entrecroisées entre maures et chrétiens, conformément au patron déjà mis en œuvre dans El Trato de Argel; de l'autre, l'aventure de Don Lope et de Zahara, dont le récit du Captif, inclus dans la première partie de Don Quichotte, nous avait offert une première version. Cette fois, les captifs chrétiens ne peuvent attendre leur salut de la décision d'un roi justicier : ils s'enfuient sur une barque que Don Lope est allé quérir à Majorque, après avoir obtenu son rachat grâce aux écus que lui a donnés Zahara. Il y a donc ici aussi une intervention de la Providence ; mais, cette fois, elle a pris pour instrument de ses desseins la fille d'Agi Morato, décidée à se convertir et à se rendre en terre chrétienne, tandis que le devenir collectif du du bagne se résorbe dans la libération d'une poignée de privilégiés. Quant à l'arrivée devant Alger d'une flotte espagnole, elle n'est plus, comme naguère, une rumeur ou un espoir incertain : c'est un simple mirage, dû aux effets du soleil levant parmi les nuées dont est chargé l'horizon. Le roi s'étonne alors de ce " prodige ", cependant que le cadi commente, non

11. Los baños de Argel, jornada 3a, vv. 2487-2489, éd. cit., p. 265.

12. Op. cit., vv. 2516-2518, p. 266.

13. Op. cit., vv. 2545-2546, p. 267. 
sans quelque ironie, le désastre qu’il a entrainé : la mort de plus de trente captifs massacrés par les janissaires, à la suite de la panique provoquée par cet événement dans la cité. Leçon désabusée qui nous donne la mesure du réajustement opéré, ainsi que du changement de perspective qui affecte la représentation du pouvoir dans l'espace de la fiction.

Le chemin parcouru par Cervantès entre les deux époques de son théâtre, en même temps qu'il révèle l'expérimentation d'une nouvelle dramaturgie, traduit une mise à distance des aspirations et des idéaux d'un temps désormais révolu. Cette évolution se trouve amplement confirmée dans le cas de La gran sultana, une pièce dont la genèse, quelque peu obscure, a laissé supposer qu' elle était le fruit d'un remaniement ${ }^{14}$ et qui a été longtemps considérée comme une turquerie, pour ne pas dire une sorte d'opéra-bouffe avant la lettre : les amours clandestines de Clara et de Lamberto, réfugiés dans le sérail, y alternent en effet avec les saillies du bouffon Madrigal pour former le contrepoint comique de l'étonnante idylle du sultan Mourad III (rebaptisé Amurat par Cervantès) ${ }^{15}$ et de Catalina de Oviedo, une jeune espagnole capturée par les Turcs alors qu' elle était enfant. S'il est vrai que cette interprétation quelque peu restrictive a été relayée récemment par des lectures plus attentives à la complexité de l'œuvre ${ }^{16}$, il n'en reste pas moins que, dans l'espace ainsi configuré, le personnage du souverain occupe cette fois une place essentielle. Dès le début de l'action, en effet, Amurat est présent sur scène : il apparaît "con mucho acompañamiento " sur le chemin de Sainte Sophie, transformée par les Turcs en mosquée, où il se rend pour y faire la prière du vendredi. La pièce s'ouvre ainsi sur une séquence spectaculaire, décrite avec force détails dans la première didascalie et dont Grecs et Turcs, au fil du dialogue, commentent le déroulement :

\section{La pompa y majestad deste tirano, sin duda alguna, sube y se engrandece sobre las fuerzas del poder humano ${ }^{17}$.}

Tel est le profil, conforme à l'image que l'on se faisait du sultan ottoman en Occident et, plus particulièrement, en Espagne ${ }^{18}$, et que va accréditer la vox populi tout au long de la première journée. Du même coup, le fait que

14. Telle est du moins l'hypothèse avancée naguère par Armando Cotarelo Valledor, pour qui La gran sultana serait une refonte de La gran turquesca, l'une des comedias de la première époque dont Cervantès donne le titre dans son Adjunta al Parnaso. Cf. «Obras perdidas de Cervantes que no se han perdido", $B R A E, \mathrm{n}^{\circ} 27,1947-1948$, p. 61-72.

15. Contemporain de Cervantès, il vécut de 1546 à 1595 et régna de 1574 jusqu’à sa mort.

16. En particulier celles que développent respectivement Ottmar Hegy (Cervantes and the Turks: Historical reality versus Literary Fiction in "La Gran Sultana» and "El amante liberal», Juan de la Cuesta Hispanic Monographs, Newark, Delaware, 1992); José Ignacio Díez (“"Sin discrepar de la verdad un punto”. La Gran Sultana: ¿Un canto a la tolerancia?», Lectura y Signo, no 1, 2006, p. 301-322) et Luis Gómez Canseco, dans l'étude préliminaire qui ouvre son excellente édition de La gran sultana, (Clásicos de Biblioteca Nueva, 66), Barcelona, Bellaterra, 2010, p. 108-128.

17. La gran sultana, jornada $1^{a}$, vv. 1-3, éd. cit., p. 373.

18. Cf. Albert Mas, Les Turcs dans la littérature espagnole au Siècle d'or, Paris, Centre de Recherches hispaniques, 1966, t. II, p. 192 et ss. 
Catalina ait vécu incognito dans le harem, six années durant, par la volonté de l'eunuque Rustán, provoque une tension qui atteint son sommet lorsqu'Amurat apprend la chose. Dès lors, au fil de ses apparitions successives, le sultan semble confirmer la réputation qui était d'emblée la sienne, en se montrant résolu à punir de mort tous ceux qui se risquent à lui désobéir : ainsi agit-il dans la première journée avec Rustán, quand il apprend l'existence de Catalina ; puis, dans la seconde, avec les deux tarasies (ou tailleurs) venus prendre les mesures de la jeune fille, lorsque celle-ci s'évanouit en découvrant que l'un d'entre eux n'est autre que son père ; enfin, dans la troisième, avec Lamberto qui, pour ne pas être démasqué, s'était travesti en femme sous le nom de Zelinda. Toutefois, à la faveur du traitement réservé à ces trois séquences, la tension chaque fois se relâche au profit d'une stylisation comique de l'épisode. La première fois, la colère du sultan tient à ce qu'en lui dépeignant Catalina comme une beauté singulière, ni Rustán, ni Mamí qui l'avait dénoncé n'ont su rendre justice à ses charmes; il faut alors que la jeune fille intercède en leur faveur pour l'apaiser. La seconde, une nouvelle intervention de Catalina permet aux deux supposés tarasies - l'autre se trouve être Madrigal- d'échapper au châtiment. La troisième, c'est encore elle qui sauve la vie de Clara et de Lamberto, sur qui le sultan avait jeté son dévolu sans s'apercevoir de son travestissement ${ }^{19}$. La tragédie imminente s'achève alors en farce : tandis que Lamberto explique qu'il doit à Mahomet d'avoir changé de sexe et s'être transformé miraculeusement en homme, Catalina, feignant un accès de jalousie, demande à Amurat de le nommer pacha de Chio.

Ébloui par les charmes de sa bien-aimée, le sultan lui accorde tout ce qu'elle lui demande. Il se voit impliqué de la sorte dans une succession d'épisodes comiques au fil desquels le prétendu "tyran " se rend aux désirs de sa captive. Cette complaisance inattendue s'appuie, selon toute vraisemblance, sur un fait corroboré par la «vérité de l'histoire », l'influence exercée sur Mourad III par deux esclaves chrétiennes : sa mère, issue d'une famille vénitienne établie à Corfou, celle des Baffo, et son épouse qui, tout comme Catalina, dansait admirablement ${ }^{20}$. Cela dit, l'ascendant de la jeune fille sur le sultan n'annule pas pour autant sa toute-puissance: il lui impose plutôt un changement de cap et de signe qui se confirme au moment où elle se dit enceinte d'un fils, futur " otomano español $\|^{21}$, projetant ainsi l'action vers une pure fantasmagorie, sous le signe de l'omnia vincit amor ${ }^{22}$.

19. «Él tiene, y en ello acierto,/ voluble la fantasía», nous dit Mamí (Op. cit., jornada 3a, vv. $2517-2518$, p. 444).

20. A. Mas, Op. cit...., t. I, p. 341-348 et plus précisément O. Hegy, Op. cit., part. p. 43-89, ainsi que Luis Gómez Canseco, éd. cit., p. 11-48.

21. C'est ainsi qu'il a été appelé par Amurat, avant même d'avoir été conçu. Cf. op. cit., jornada $1^{a}$, vv. 1217, p. 407.

22. La convergence des destins respectifs du Grand Turc et de la jeune espagnole se dessine en effet comme une utopie, soit qu'elle s'inscrive dans un contexte diplomatique qui la dément, celui d'un abandon par la monarchie espagnole d'une véritable politique méditerranéenne, au bénéfice d'un vague rêve d'alliance avec la Perse. (Cf. à ce sujet notre Cervantès dramaturge, Paris, 
Ces remodelages successifs marquent, par conséquent, les étapes d'une trajectoire qui trouve un aboutissement pour le moins inattendu dans Pedro de Urdemalas. Dernière des œuvres qui composent le recueil des Ocho Comedias, cette pièce nous offre, dans une atmosphère de pure fantaisie, le nec plus ultra de la réélaboration cervantine de la figure du roi. Il n'y a plus, cette fois, de référent historique auquel renvoie le personnage. Issue d'un folklore dont procède directement Pedro, le protagoniste qui donne son titre à la pièce ${ }^{23}$, l'action se situe dans une totale uchronie. Du monarque anonyme qui apparait sur scène, au milieu de la deuxième journée, rien ou presque ne nous est dit; nous apprenons seulement que son frère, bras armé d'une Reconquête mythique, se bat depuis plusieurs années sur les frontières, «do apoca»

$$
\text { con su lanza la morisma }{ }^{24} \text {. }
$$

Ce qui justifie son intervention, c'est le rôle qui lui est accordé au sein de l'intrigue secondaire, animée par la gitane Belica, proche parente de Preciosa, l'héroïne de La Gitanilla. À l'occasion d'une chasse, il fait la rencontre de la jeune fille et s'en éprend, provoquant du même coup la jalousie et la fureur de son épouse. Belica, pour sa part, voit dans cette rencontre un signe du destin : née dans des circonstances qui s'éclairent au début de la troisième journée - elle est le fruit des amours secrètes d'une duchesse et de ce frère dont il a été question plus haut -, elle a été confiée à une gitane qui l'a élevée. Le monarque se trouve du même coup dans une situation éminemment ambiguë; car si la reine semble s'apaiser en apprenant de qui elle est la fille, Belica devient pour le roi un fruit défendu que la parenté qui les unit met désormais à sa portée, mais que l'interdit de l'inceste rend irrémédiablement inaccessible. Autant dire que, si sa légitimité n'est pas remise en cause, l'autorité dont il est investi n'en apparaît pas moins dérisoire.

À la faveur de la réunion finale des trajectoires respectives de Pedro et de Belica, le dénouement s'ouvre sur un autre horizon que celui qu'impliquent habituellement les conventions d'une simple anagnorisis. Tandis que la jeune gitane voit s'accomplir le destin auquel elle s'est toujours crue promise, Pedro de Urdemalas, sur un tout autre registre, vit une métamorphose parallèle à la sienne. "Hijo de la piedra " ${ }^{25}$ et, par là même, fils de ses œuvres, il s'en va rejoindre une troupe de comédiens qui se préparent à jouer devant Leurs Majestés. Il en vient ainsi à inscrire le théâtre au sein du théâtre, mais dans un

PUF, 1977, p. 397-398), soit qu'elle renvoie, sub specie recreationis, au problème de l'impossible coexistence entre morisques et vieux-chrétiens dans la péninsule (comme le veut Francisco Márquez Villanueva, dans Moros, moriscos y turcos en Cervantes, Barcelona, Bellaterra, 2010, p. 187-214). Pour Luis Gómez Canseco (éd. cit., p. 108-128), Catalina, confrontée à une alternative entre deux péchés mortels (l'union avec un infidèle ou le suicide), choisirait la voie du probabilisme, dans le cadre ambigu d'une tragédie inachevée qui se résoudrait dans une fête en demi-teinte.

23. Cf. Cervantès dramaturge, p. 125-128.

24. Pedro de Urdemalas, jornada 3a, vv. 2501-2502, éd. cit., p. 702.

25. Ibid., jornada $1^{\text {a }}$, v. 600 , p. 650. 
rapport de contiguïté distinct du traditionnel dédoublement de plans que ce procédé détermine habituellement. Ainsi qu'il le dit à Belica :

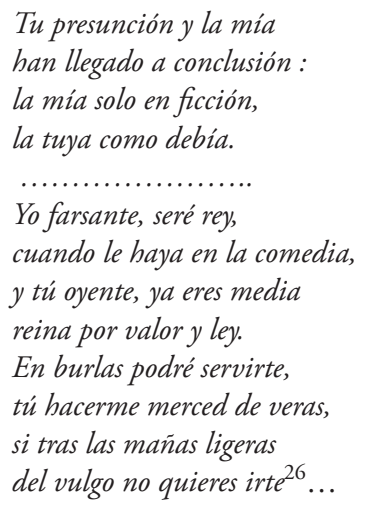

Devenu roi dans la pièce dont la représentation s'avère imminente, il se dispose ainsi à triompher sur une autre scène que celle de la comedia qui s'achève sous nos yeux : une scène virtuelle qui, conformément à l'image bien connue du theatrum mundi, nous apparaît comme l'analogon du monde où nous vivons.

À l'heure où se conclut l'action, Pedro contemple donc son propre personnage, "hecho [...] una quimera ${ }^{27}$ : il reçoit les attributs dérisoires que lui confère le don de la parole, dans un théâtre renvoyé dans les coulisses et qui s'évanouit au moment même où il allait naître. Dans ces conditions, ce nouveau mirage s'avère être le chant du cygne d'une dramaturgie expérimentale, dans une ultime tentative pour incarner sur scène le personnage emblématique du roi : un personnage désacralisé qui, même s'il se dissout à la dernière minute dans un futur brumeux, ne laisse pas moins de s'imposer comme une pièce fondamentale de ce jeu.

26. Ibid., jornada 3a, vv. 3032-3035 et 3040-3047, p. 716. Sur l'insertion du théâtre dans le théâtre, cf. Cervantès dramaturge, p. 366-377.

27. Ibid.., v. 3142, p. 718. 